\title{
Micro-Hall bar as a sensor to detect the interaction of nanoscale ferromagnetic disks and columns
}

\author{
Y. L. Hao and F. M. Peeters ${ }^{\text {a) }}$ \\ Department of Physics, University of Antwerpen, Groenenborgerlaan 171, B-2020 Antwerpen, Belgium
}

(Received 26 February 2007; accepted 17 April 2007; published online 29 June 2007)

\begin{abstract}
The response of a ballistic two-dimensional electron gas (2DEG) confined to a Hall cross and subjected to a locally inhomogeneous magnetic field which is created by two parallel or antiparallel magnetized cylindrical dots deposited above the 2DEG is investigated. The Hall and bend resistances are calculated as a function of the magnetization of the magnetic dots for different positions of the dots on the Hall bar. The Hall and bend resistances are different for parallel and antiparallel magnetized dots and depend on the relative position of the magnetic dots on the Hall bar. (C) 2007 American Institute of Physics. [DOI: 10.1063/1.2743880]
\end{abstract}

\section{INTRODUCTION}

Driven by the enthusiasm of possible applications in high-density storage media, more and more research activities are directed toward nanometer-scale magnetic particles. Using scanning tunneling microscope-induced micro-organic vapor deposition techniques (STM-MOCVD), the diameter of the magnetic particles can be as small as $5 \mathrm{~nm}$. But, another fact restricts the achievable density of magnetic recording: the interaction between the particles, which becomes more and more important when the particles get smaller and closer to each other. Due to the interaction, the adjacent magnetic particles will favor an antiparallel magnetization orientation, ${ }^{1}$ but in the presence of an external field a parallel magnetization orientation is expected. Here, we provide theoretical evidence that a Hall bar in the ballistic regime can be used as a noninvasive tool to identify the orientation of magnetization of the adjacent particles and therefore give us important information on the interparticle magnetic interaction.

Geim and co-workers ${ }^{2,3}$ investigated the case where there is only one magnetic particle at the center of the Hall cross. They found that the Hall resistance is suppressed when the particle has a high magnetization; and for low magnetization the Hall resistance is completely determined by the average field in the cross region, as predicted in Ref. 4 when the Hall bar is in the ballistic regime. In the diffusive regime ${ }^{5}$ the active region is about twice the Hall cross area.

Hysteresis traces of individual magnetic nanodisks featuring a magnetic vortex were obtained using the above micro-Hall magnetometry. ${ }^{6,7}$ Vortex pinning and switching on individual nanodisks containing nanoholes was investigated recently ${ }^{8}$ using the same measuring technique.

In this article, we study the situation when more than one magnetic particle is present in the cross region and calculate the response of the Hall bar, i.e., the Hall and the bend resistance. From these resistances we are able to determine the relative magnetization direction of the magnetic elements giving information on their exchange interaction. The billiard model $^{9}$ is used to simulate the electron motion inside the

${ }^{a)}$ Electronic mail: francois.peeters@ua.ac.be
Hall cross, and we calculate the Hall and bend resistances using the Landauer-Büttiker formula ${ }^{10,11}$ for different values of the magnetization of the two magnets and different positions of such magnets in the Hall cross.

The article is organized as follows. In Sec. II we present our theoretical approach. The numerical results are given in Sec. III and our conclusions in Sec. IV.

\section{THEORETICAL MODEL}

The system we investigate is shown in Fig. 1(a). It is a Hall cross with four identical leads; each of them is $2 w$ $=1.85 \mu \mathrm{m}$ wide (the parameters are in accordance with Ref. 2). Cylindrical magnetic dots are put on the surface of the cross, which are parallel or antiparallel magnetized. The field created by the dots will influence the two-dimensional electron gas (2DEG) that is positioned $70 \mathrm{~nm}$ below the surface with a density $n_{e}=3.45 \times 10^{15} \mathrm{~m}^{-2}$ [see Figs. $1(\mathrm{c})$ and $1(\mathrm{~d})$ ], and result in a change of the Hall and bend resistances.
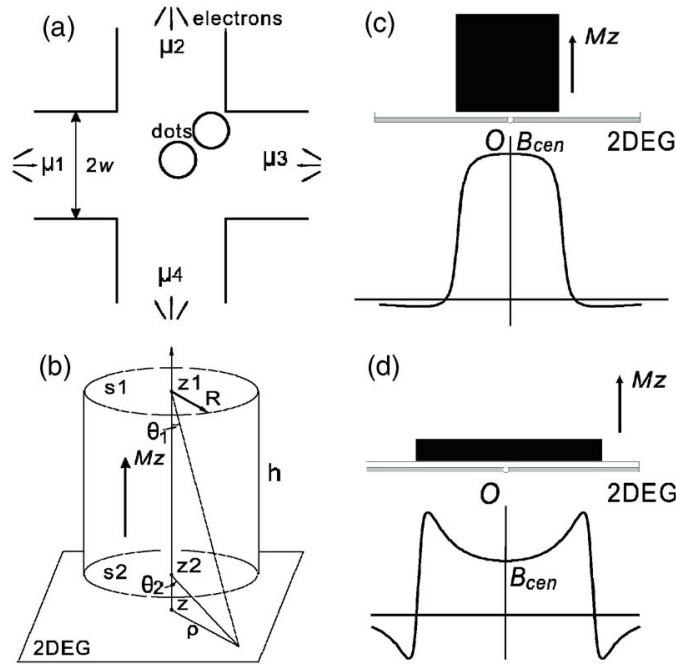

FIG. 1. (a) Schematic diagram of the systems to be investigated: Ballistic electrons are injected into a Hall cross in the presence of two ferromagnetic dots which are put on top of the 2DEG. (b) The cylindrical ferromagnetic column with the relevant geometrical parameters. Magnetic field profile created by a (c) thick and (d) thin dot deposited above the 2DEG. 
First we calculate the magnetic field profile of a magnetic cylinder. Note that the cylinder has an azimuthal symmetric magnetic field profile, and a general solution for such a problem is ${ }^{12}$

$$
\Phi(r, \theta)=\sum_{l=0}^{\infty}\left[A_{l} r^{l}+B_{l} r^{-(l+1)}\right] P_{l}(\cos \theta),
$$

where $A_{l}$ and $B_{l}$ are the expansion coefficients, and $P_{l}(\cos \theta)$ is the Legendre polynomial. The magnetic field along the symmetry axis of the cylinder [see Fig. 1(b)] is $B_{z}(z)=$ $\left(\mu_{0} M_{z} / 2\right)\left[\left(z-z_{1}\right) / \sqrt{R^{2}+\left(z-z_{1}\right)^{2}}-\left(z-z_{2}\right) / \sqrt{R^{2}+\left(z-z_{2}\right)^{2}}\right]$. We expand this expression into a power series, and compare its coefficients to the general solution Eq. (1), from which we obtain the coefficients $A_{l}$ and $B_{l}$. The resulting magnetic field of a cylinder is

$$
B_{z}=\frac{\mu_{0} M_{z}}{2}\left[F\left(r_{1}, \cos \theta_{1}\right)-F\left(r_{2}, \cos \theta_{2}\right)\right],
$$

where $r_{i}=\sqrt{\left(z-z_{i}\right)^{2}+\rho^{2}}$ and $\cos \theta_{i}=\left(z-z_{i}\right) / \sqrt{\left(z-z_{i}\right)^{2}+\rho^{2}}$, with $F\left(r_{i}, \cos \theta_{i}\right)=$

$$
\begin{array}{ll}
\sum_{n=0}^{\infty} P_{2 n}(0)\left(\frac{r_{i}}{R}\right)^{2 n+1} P_{2 n+1}\left(\cos \theta_{i}\right), & 0 \leq r_{i} \leq R, \\
1+\sum_{n=1}^{\infty} P_{2 n}(0)\left(\frac{R}{r_{i}}\right)^{2 n} P_{2 n-1}\left(\cos \theta_{i}\right), & r_{i} \geq R .
\end{array}
$$

Following Ref. 11 for a four-terminal device like a Hall cross, we assume that the current flows from lead $m$ to lead $n$, and the voltage is measured between leads $k$ and $l$. The corresponding resistance can be written as

$$
R_{m n, k l}=\frac{h}{2 e^{2}} \frac{T_{k m} T_{l n}-T_{k n} T_{l m}}{D},
$$

where $T_{i j}$ is the electron transmission probability from lead $i$ to lead $j$, which is determined by using the billiard model introduced in Ref. 9 and $D$ is the subdeterminant of the matrix of all the transmission probabilities. ${ }^{11,13}$ In this article, we took $R_{H}=R_{13,24}$ as the Hall resistance. For the bend resistance, which is defined as the ratio between the voltage measured between two adjacent leads and the current put through the opposite pair of leads, we took $R_{B}=R_{14,32}$. The 2DEG is assumed to be in the ballistic regime (which implies a high mobility sample with $\ell_{e}>2 w$, where $\ell_{e}$ is the mean free path of the 2DEG). In the billiard model, the electrons are injected into the cross region with velocity equal to the Fermi velocity and different directions. The motion of these "classical" electrons is governed by Newton's second law and experiences a nonhomogeneous magnetic field profile from the magnetic dots and specular reflected by the boundary of the cross. Quantum effects are assumed to be negligible, which is valid when temperature is not too small.

\section{RESULTS}

We found a symmetry property of the Hall resistance: when an axis-symmetry magnetic field (ASMF) is present with its symmetry axis transverse or longitudinal to the axis of the Hall cross, and for the same distance to the center of

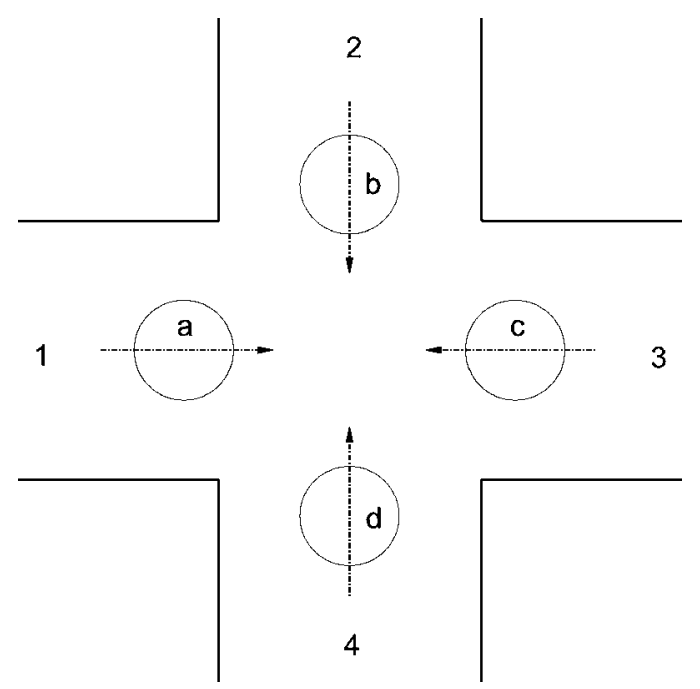

FIG. 2. A ferromagnetic round disk (diameter $=0.75 w$ ), which produces an axis-symmetry magnetic field (ASMF) in the 2DEG (its symmetry axis as shown in the figure), is put with its center at: (a) $(-1.25 w, 0)$; (b) $(0,1.25 w)$; (c) $(1.25 w, 0)$; and (d) $(0,-1.25 w)$, which leads to the same Hall resistance at all four of these positions.

the Hall cross, then there are four possible positions (for example, see Fig. 2), such that the Hall resistance will be the same for all these positions of the ASMF.

We restrict ourselves to two identical ferromagnetic dots $(r=0.65 \mu \mathrm{m}, h=1.5 \mu \mathrm{m})$ with parallel magnetization (PM, see Fig. 3) or antiparallel magnetization (APM, see Fig. 4). We calculated the Hall and bend resistances for different relative positions of the magnetic dots above the Hall cross. The first dot which is kept fixed is put with its center at $(-w$, $0)$ and the second at: (a) $(0, w)$; (b) $(w, 0)$; and (c) $(0,-w)$ [see the inset of Fig. 3(a)]. The magnetization of each dot increased from zero to $10^{6} \mathrm{~A} / \mathrm{m}$, which yields a maximum field strength of about $0.53 \mathrm{~T}$ below the dot.

If these are two PM dots, the resistances are expressed as a function of $B_{\mathrm{av}}$, which is the average field in the cross region. We know from Ref. 4 that the Hall resistance will only depend on $B_{\text {av }}$ in the low field regime. Our results are in good agreement with this conclusion, as shown in Fig. 3(a). When the magnetization increases, the Hall resistance becomes very sensitive to the position of the second cylinder; when it is placed at position (c) it will yield a much higher $R_{H}$ than when it is placed at position (a). This is different from the one-cylinder case, where the Hall resistance was found to be larger when we put the cylinder at position (a). In Figs. 3(a) and 3(b), we show also the $R_{H}$ and $R_{B}$ in the case where a uniform magnetic field is applied and no dots are present. If the two dots are APM, because the average field is zero, we show the resistances as function of $B_{\text {cen }}$, which is the field strength at the center of the dot in the 2DEG plane, as point $O$ in Figs. 1(c) and 1(d). From Ref. 4 the Hall resistance should be zero at low fields. We found that this is only true for very small fields in the case where the second dot is placed at positions (a) and (c), while for dot position (b) it is valid for arbitrary value of the magnetization. Notice that curves (a) and (c) exhibit an interesting sign reversal. 

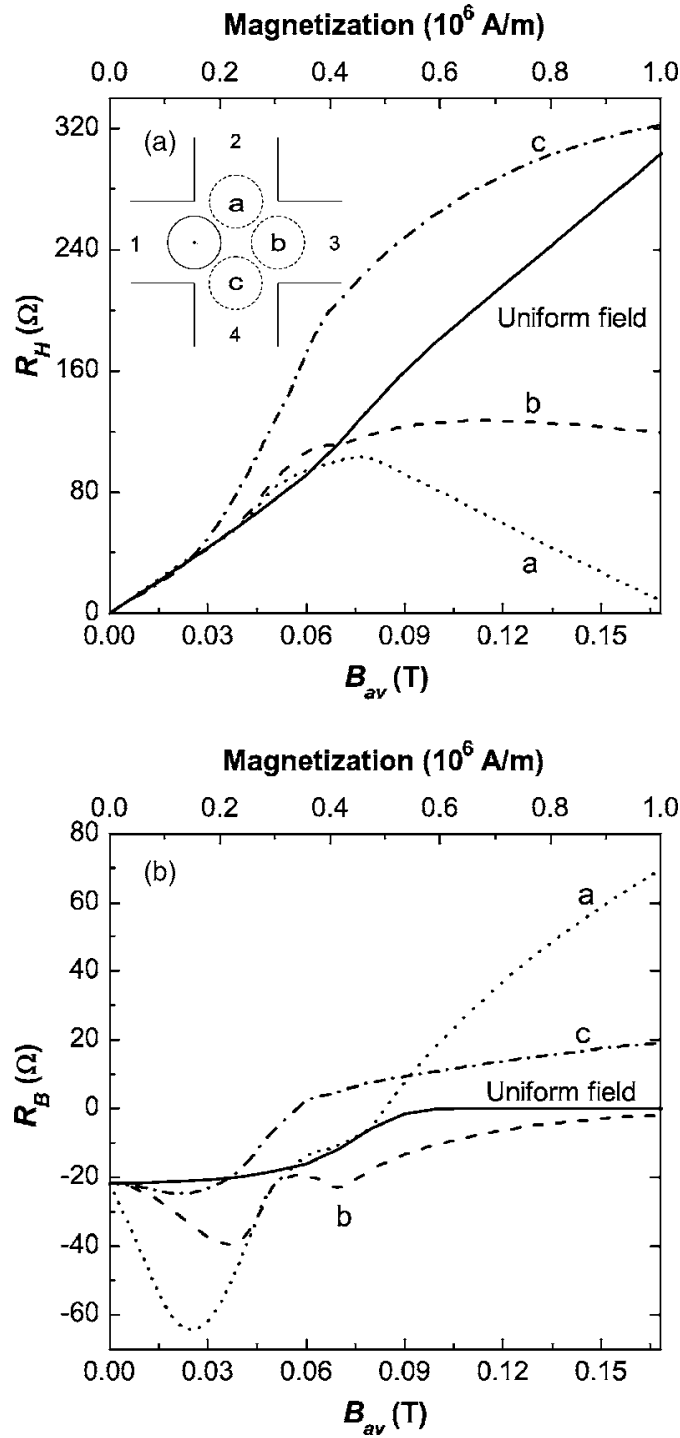

FIG. 3. (a) The Hall resistance $R_{H}$ for two parallel magnetized dots as a function of the magnetization of the dots or as a function of the average field $B_{\text {av }}$ in the cross region; (b) The corresponding bend resistance $R_{B}$ for these two dots. The different curves correspond to different positions of one of the magnetic dots [see the inset of (a)].

The difference in the Hall resistance for PM and APM dots can be summarized as follows:

(1) If the second dot is placed at position (a), at low magnetization, we found that the difference in the Hall resistance between PM and APM dots is maximum (see Fig. 5), since the Hall resistance of the PM dots is positive and increases and that of the APM dots is negative and decreases with increasing magnetization. For intermediate magnetization the Hall resistances of the APM dots and the PM dots both increase up to a point where a maximum is reached and they start to decrease for high magnetization. The PM Hall resistance is always larger than the APM one; the smallest difference occurs for $M_{z}=6 \times 10^{5} \mathrm{~A} / \mathrm{m}$ and is about $22.7 \Omega$.

(2) If the second dot is placed at position (b), the Hall resistance will always be zero for the APM dots, which is a consequence of the symmetric electron trajectories due to the antisymmetric magnetic field. For the PM dots,
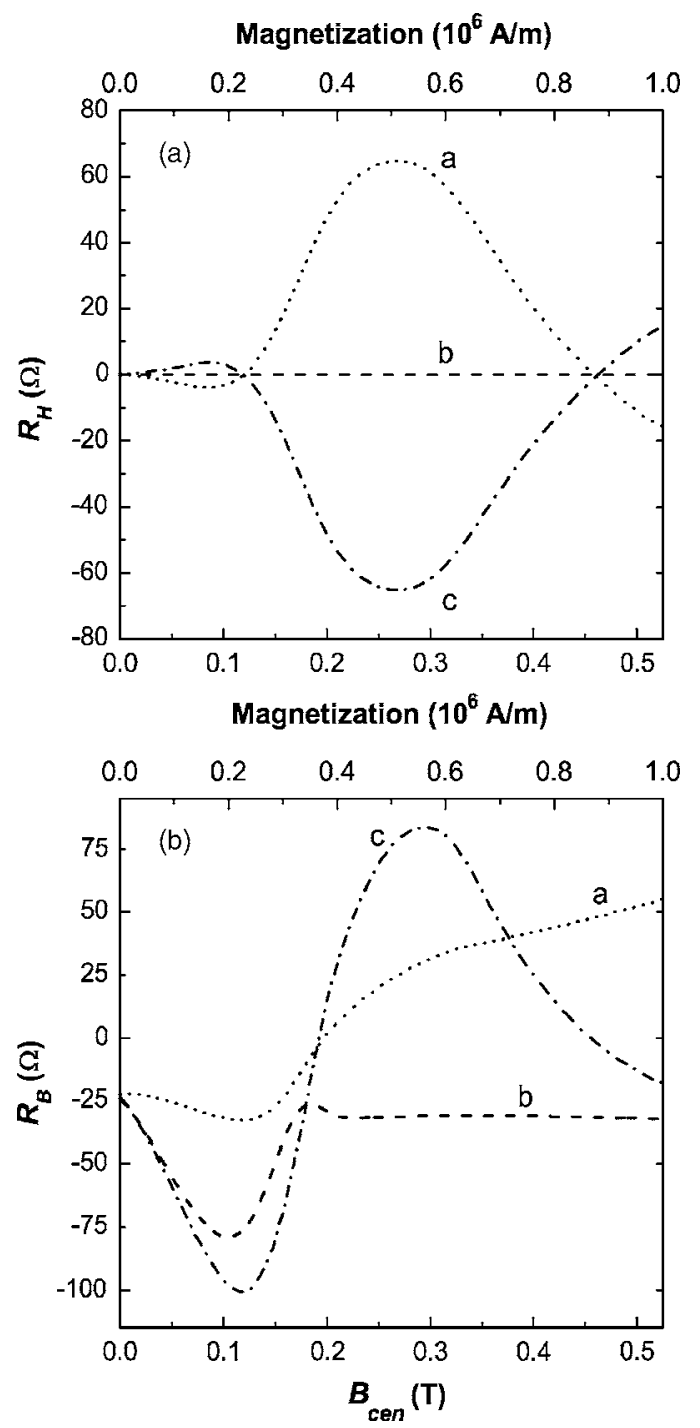

FIG. 4. The same as Fig. 3 but now the second dot has a magnetic moment opposite to the first one. The resistances are now plotted as function of $B_{\text {cen }}$ $\left(B_{\mathrm{av}}=0\right.$ here $)$.

the Hall resistance increases and saturates to about $120.0 \Omega$ [see Fig. 3(a)].

(3) If the second dot is placed at position (c), the difference in the Hall resistance at low magnetization is smallest,

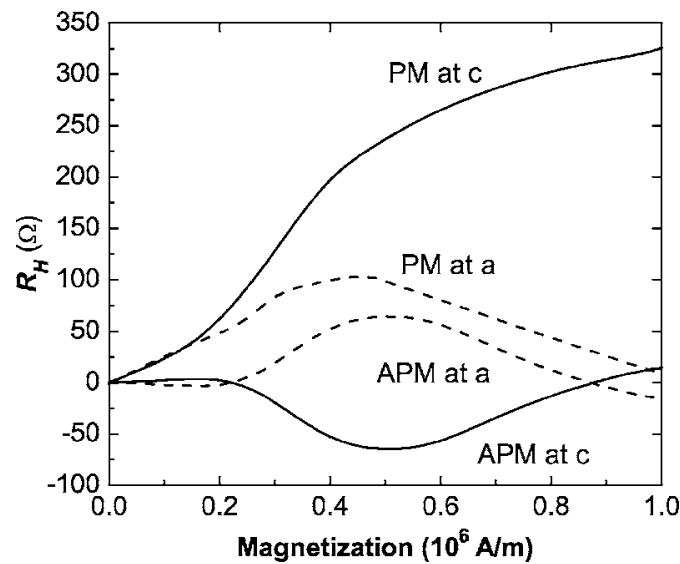

FIG. 5. Comparison of the Hall resistance in the case of PM dots and APM dots: dashed lines, the second dot placed at position (a); solid lines, the second dot placed at position (c). 
since the APM dots also have an increased positive Hall resistance as the PM dots (see Fig. 5). But, at intermediate magnetization, the difference in the Hall resistance become very large, because $R_{H}$ of the APM dots decreases to negative values, while that of the PM dots increases very fast. In fact, $R_{H}(a)=-R_{H}(c)$ for the APM configuration.

The bend resistances are sensitive to the details of the magnetic field profile, and depend very much on the position of the magnetic disks on the Hall cross [see Fig. 3(b) and Fig. 4(b)]. Notice that, while the corresponding Hall resistances are the same in the small magnetic field regime [see Fig. $3(\mathrm{a})$ ], the corresponding bend resistances can be rather different. This implies that the bend resistance is a more sensitive quantity than the Hall resistance to detect the magnetic field profile in the low field regime.

\section{CONCLUSIONS}

Our study shows that the ballistic micron-sized Hall bar can be used as a noninvasive tool to detect the relative direction of the magnetization of ferromagnetic nanoparticles (see Fig. 5). The Hall resistance for two PM dots is always larger than that for two APM dots. The size of the difference depends on the relative position of the dots. In low magnetization of the dots the bend resistance is a more sensitive quantity than the Hall resistance for the relative position of the magnetic dots.
In our calculation we restricted ourselves to identical ferromagnetic dots that have the same magnitude of magnetization. It is straightforward to generalize the present result to nonidentical dots and to cases in which the magnitude of their magnetization is different.

\section{ACKNOWLEDGMENTS}

This work is supported by the Belgian Science Policy (IUAP), the Flemish Science Foundation (FWO-VI), and the Chinese-Flemish bilateral program.

${ }^{1}$ S. Wirth and S. von Molnár, J. Appl. Phys. 87, 7010 (2000).

${ }^{2}$ K. S. Novoselov, A. K. Geim, S. V. Dubonos, Y. G. Cornelissens, F. M. Peeters, and J. C. Maan, Phys. Rev. B 65, 233312 (2002).

${ }^{3}$ A. K. Geim, S. V. Dubonos, J. G. S. Lok, I. V. Grigorieva, J. C. Maan, L. Theil Hansen, and P. E. Lindelof, Appl. Phys. Lett. 71, 2379 (1997).

${ }^{4}$ F. M. Peeters and X. Q. Li, Appl. Phys. Lett. 72, 572 (1998).

${ }^{5}$ I. S. Ibrahim, V. A. Schweigert, and F. M. Peeters, Phys. Rev. B 57, 15416 (1998).

${ }^{6}$ T. M. Hengstmann, D. Grundler, Ch. Heyn, and D. Heitmann, J. Appl. Phys. 90, 6542 (2001).

${ }^{7}$ M. Rahm, M. Schneider, J. Biberger, R. Pulwey, J. Zweck, and D. Weiss, Appl. Phys. Lett. 82, 4110 (2003).

${ }^{8}$ M. Rahm, J. Stahl, W. Wegscheider, and D. Weiss, Appl. Phys. Lett. 85, 1553 (2004).

${ }^{9}$ C. W. J. Beenakker and H. van Houten, Phys. Rev. Lett. 63, 1857 (1989).

${ }^{10}$ R. Landauer, IBM J. Res. Dev. 1, 233 (1957).

${ }^{11}$ M. Büttiker, Phys. Rev. Lett. 57, 1761 (1986).

${ }^{12}$ J. D. Jackson, Classical Electrodynamics, 3rd ed. (Wiley, New York, 1998), p. 101

${ }^{13}$ K. L. Shepard, M. L. Roukes, and B. P. Van der Gaag, Phys. Rev. Lett. 68 , 2660 (1992). 